\title{
Recurrent overdose: patient characteristics, habits, and outcomes
}

\author{
David McD Taylor, Peter A Cameron, David Eddey
}

\begin{abstract}
Objectives-Patients who overdose repeatedly on drugs and poisons (repeaters) are of major concern. This study aimed to examine the demographics, types of drugs ingested, associated self inflicted trauma, and medium term outcome of repeaters and to compare these patients with those who overdosed on one occasion only (single presenters) during the study period.
\end{abstract}

Methods-The study was undertaken in the emergency department of a large, provincial Australian hospital. A retrospective case note examination was made for all patients who presented, after drug overdose, during the two year study period. These patients were also followed up for a further 12 months after the study period.

Results-The study identified 335 single presenters and 46 repeaters. Females formed about two thirds of each group but repeaters tended to be older $(p>0.05)$ and to present more frequently before midnight $(p>0.05)$. Significantly more repeat presentations were triaged to the low priority categories 4 or 5 (odds ratio (OR) $0.48 ; 95 \%$ confidence interval (CI) 0.26 to $0.90, p=0.023$ ) and this group required fewer admissions to the hospital (OR 1.85; 95\% CI 1.16 to $2.93, p=0.009)$. Repeaters tended to take single drug overdoses. There were significantly more paracetamol only overdoses (OR 0.54; 95\% CI 0.32 to $0.92, p=0.024$ ) and neuroleptic only overdoses (OR 0.27 ; 95\% CI 0.11 to 0.67 , $\mathbf{p}=\mathbf{0 . 0 0 5}$ ) in the repeater group. More repeaters caused self inflicted trauma during the study period (OR $0.20 ; 95 \%$ CI 0.06 to $0.64, p=0.007$ ). No repeater completed suicide during the study or the 12 month follow up periods but repeaters presented more frequently, after overdose, during follow up (OR 0.38; 95\% CI 0.13 to $1.11, p=0.078$ ).

Conclusions-The study concludes that there are some significant differences between patients who overdose repeatedly and those who overdose on one occasion only. The study findings suggest that the medium term suicidal risk for repeaters is relatively low. However, this risk will vary and individual patients must be assessed thoroughly and managed accordingly.

( 7 Accid Emerg Med 1998;15:257-261)

Keywords: overdose; parasuicide; suicide; toxicology
The number of young Australians, especially men, who commit suicide has risen over the past 15 years and is now among the highest in the industrialised world. ${ }^{1-4}$ Intentional overdose of medicinal drugs and toxins is a common cause of completed suicide generally and the commonest cause in women. ${ }^{1}$ Nonfatal overdose is seen commonly and emergency department presentations have been estimated at 7500-10000 per year in the state of Victoria alone. ${ }^{5}$

Overdose is frequently seen in patients with psychiatric illness, ${ }^{23-9}$ especially depression, and may be associated with significant morbidity and mortality. ${ }^{1-3} 78$ It may be associated with overt suicidal ideation ${ }^{389}$ or the patient may be seeking attention or secondary gain. ${ }^{9} 10$ It is not uncommon for some patients to present repeatedly after overdose. Emergency department staff may find these "repeaters" difficult to manage and often have negative attitudes towards them. ${ }^{11}{ }^{12}$ Some investigators have found that these patients less frequently take dangerous overdoses ${ }^{13}$ and tend to have more social and personality problems than significant depressive illness. ${ }^{13}{ }^{14}$ To date, little has been done in Australia to investigate the characteristics of patients who overdose repeatedly.

This study aimed to examine the population demographics, the types of drugs ingested, the associated incidence of self inflicted trauma, and the medium term outcome of the group of patients who overdosed on more than one occasion during the study period. It aimed to compare this group of repeaters with those patients who presented after overdose on only one occasion.

\section{Methods}

Geelong Hospital, Victoria, Australia, is a 450 bed community teaching hospital which serves a population of approximately 250000 residents covering urban and rural areas. It is the only major hospital in the area and is well suited to epidemiological studies of this type. A retrospective analysis of the emergency department computerised records was undertaken for the two year study period of 1 January 1993 to 31 December 1994, inclusive. All records where the attending doctor diagnosed overdose or other self inflicted injury were identified. The records provided personal details, the triage category, a description of drugs ingested (determined by history or laboratory assay) or injury sustained, patient management, disposal, and arrangements for follow up for each patient. The minimum data set used in this study coded a diagnosis of drug overdose as an injury. Previous analyses of the quality of 
Table 1 Age and sex of single presenters and repeat presenters in 1993-94 (\% in parentheses)

\begin{tabular}{llrllllll}
\hline \multicolumn{7}{l}{ Age group (years) } \\
\cline { 2 - 7 } & $<15$ & $15-24$ & $25-34$ & $35-44$ & $45-54$ & $55-64$ & $\geqslant 65$ & Totals \\
\hline Single presenters & & & & & & & & \\
$\quad$ Female & $3(0.9)$ & $95(28.4)$ & $63(18.8)$ & $30(9.0)$ & $17(5.1)$ & $7(2.1)$ & $10(3.0)$ & $225(67.2)$ \\
$\quad$ Male & 0 & $37(11.0)$ & $29(8.7)$ & $28(8.4)$ & $9(2.7)$ & 0 & $7(2.1)$ & $110(32.8$ \\
Total & $3(0.9)$ & $132(39.4)$ & $92(27.5)$ & $58(17.3)$ & $26(7.8)$ & $7(2.1)$ & $17(5.1)$ & $335(100)$ \\
Repeat presenters & $1(2.2)$ & $10(21.7)$ & $9(19.6)$ & $6(13.0)$ & $1(2.2)$ & $3(6.5)$ & $1(2.2)$ & $31(67.4)$ \\
$\quad$ Female & 0 & $5(10.9)$ & $10(21.7)$ & 0 & 0 & 0 & 0 & $15(32.6)$ \\
$\quad$ Male & $1(2.2)$ & $15(32.6)$ & $19(41.3)$ & $6(13.0)$ & $1(2.2)$ & $3(6.5)$ & $1(2.2)$ & $46(100)$ \\
Total & & & & & & & &
\end{tabular}

coding of this injury diagnosis data have demonstrated greater than $95 \%$ accuracy. ${ }^{15}$

In this study, a "single presenter" was defined as a patient who made a "single presentation" only, after overdose, during the study period. This is regardless of whether the patient had ever presented, after overdose, before the study period. A "repeater" was defined as a patient who made two or more presentations, after overdose, during the study period. "Repeat presentations" made by the repeaters included their first and subsequent presentations after overdose. The "general patient" group included all presentations to the emergency department during the study period.

The study included all patients who presented alive to the emergency department after overdose or self inflicted injury. Patients who died before reaching hospital were not entered onto the emergency department computerised record. Therefore, it was not possible to determine if any known single presenters or repeaters died outside of hospital during or after the study period. The triage categories were based on the national triage scale ${ }^{16}$ and indicate the recommended times within which the patient should be seen. Patients were allocated a triage category of 1 to 5 indicating that their longest waiting times should be $0,10,30,60$, or 120 minutes, respectively.

Various methods were used to determine whether the repeaters from the 1993-94 study period were still alive after a further 12 months. The hospital emergency department and Victorian psychiatric services computerised records identified those patients who presented for treatment after 1 January 1996. These patients must therefore have been alive at the end of the 12 month follow up. For each remaining patient, telephone contact with the patient, their family or general practitioner, after 1 January 1996, determined their survival status.

Odds ratio (OR) analysis was used when comparing proportions of various indices within two population groups. The significance ( $p$ value) of these ORs was determined by the exclusion (significant) or inclusion (not significant) of 1 within the $95 \%$ confidence interval (CI).

\section{Results}

During the study period, there were 67048 general patient presentations. Of these, 441 $(0.66 \%)$ presentations occurred after overdose. There were 335 single presenters and 46 repeaters. The repeaters made a total of 106 presentations after overdose (mean $=2.3$ presentations per repeater).

Table 1 describes the age and sex of the overdose patients. Females were more commonly seen and formed about two thirds of each group. The largest age groups of single presenters and repeaters were 15-24 years and 25-34 years, respectively. Despite the different proportions of patients within each age group, none was statistically significant $(p>0.05)$.

Presentations were most frequent in the late afternoon and evening. There were $69(20.6 \%)$ and $96(28.7 \%)$ single presentations between 1600-1959 and 2000-2359 hours, respectively. There were $30(28.3 \%)$ and $33(31.1 \%)$ repeat presentations during the same periods, respectively. Single presentations tended to be more evenly distributed throughout the day. Between 0000-0800 hours, there were 69 $(20.6 \%)$ single but only $14(13.2 \%)$ repeat presentations. Despite the different proportions of presentations within each time bracket, none was statistically significant $(p>0.05)$. Almost half of the presentations from both groups were early in the working week. There were $165(49.3 \%)$ single and $50(47.2 \%)$ repeat presentations on either Monday, Tuesday, or Wednesday ( $p>0.05$ ). All other presentations were distributed evenly over the other days of the week. Presentations from both groups were distributed evenly throughout the months of the year.

Table 2 describes the triage categorisation of presentations after overdose. There was a significantly greater proportion of single (OR $4.47 ; 95 \%$ CI 3.57 to $5.61, \mathrm{p}<0.001$ ) and repeat (OR $3.30 ; 95 \%$ CI 2.16 to 5.04 , $\mathrm{p}<0.001)$ presentations triaged as categories 1

Table 2 Triage categories of single presenters, repeat presenters, and general patient presentations in $1993-94(\%$ in parentheses)

\begin{tabular}{|c|c|c|c|c|c|c|}
\hline & \multicolumn{5}{|c|}{ Triage category } & \multirow[b]{2}{*}{ Totals } \\
\hline & 1 & 2 & 3 & 4 & 5 & \\
\hline Single presenters & $12(3.6)$ & $104(31.0)$ & $189(56.4)$ & $27(8.1)$ & $3(0.9)$ & $335(100)$ \\
\hline Repeat presenters & 0 & $30(28.3)$ & $58(54.7)$ & $16(15.1)$ & $2(1.9)$ & $106(100)$ \\
\hline General patients & $604(0.9)$ & $6575(9.8)$ & $26675(39.8)$ & $27606(41.1)$ & $5588(8.4)$ & $67048(100)$ \\
\hline
\end{tabular}


Table 3 Disposition of single presenters $(n=335)$ and repeat presenters $(n=106)$ (\% in parentheses)

\begin{tabular}{|c|c|c|c|c|c|c|c|c|c|c|}
\hline & \multicolumn{5}{|l|}{ Admitted } & \multicolumn{5}{|l|}{ Discharged } \\
\hline & Ward & $\begin{array}{l}\text { Intensive } \\
\text { care }\end{array}$ & $\begin{array}{l}\text { Emergency } \\
\text { department }\end{array}$ & $\begin{array}{l}\text { Psychiatric } \\
\text { ward }\end{array}$ & Transferred & GP review & $\begin{array}{l}\text { Home, no } \\
\text { review }\end{array}$ & $\begin{array}{l}\text { Psychiatric } \\
\text { outpatients }\end{array}$ & $\begin{array}{l}\text { Private } \\
\text { psychiatrist }\end{array}$ & Other \\
\hline Single presenters & $116(34.6)$ & $40(11.9)$ & $27(8.1)$ & $23(6.9)$ & $2(0.6)$ & $39(11.6)$ & $24(7.2)$ & $20(6.0)$ & $8(2.4)$ & $36(10.8)$ \\
\hline Repeat presenters & $29(27.4)$ & $5(4.7)$ & $13(12.3)$ & $9(8.5)$ & $1(0.9)$ & $11(10.4)$ & $4(3.8)$ & $17(16.0)$ & $4(3.8)$ & $13(12.2)$ \\
\hline
\end{tabular}

^Includes other destinations, left against advice, did not wait.

Table 4 Description of drugs ingested by single presenters and repeaters in 1993-94 (\% in parentheses)

\begin{tabular}{|c|c|c|c|c|c|c|}
\hline \multirow[b]{2}{*}{ Drug class } & \multicolumn{3}{|c|}{ Single presenters } & \multicolumn{3}{|c|}{ Repeat presenters } \\
\hline & As sole drug & In combination & Total & As sole drug & In combination & Total \\
\hline Benzodiazepine & $81(17.8)$ & $45(9.9)$ & $126(27.6)$ & $26(19.7)$ & $10(7.6)$ & $36(27.3)$ \\
\hline Paracetamol & $49(10.7)$ & $30(6.6)$ & $79(17.3)$ & $24(18.2)$ & $4(3.0)$ & $28(21.2)$ \\
\hline Antidepressants & $47(10.3)$ & $25(5.5)$ & $72(15.8)$ & $8(6.1)$ & $10(7.6)$ & $18(13.6)$ \\
\hline Neuroleptics & $10(2.2)$ & $12(2.6)$ & $22(4.8)$ & $10(7.6)$ & $6(4.5)$ & $16(12.1)$ \\
\hline Opioids & $2(0.4)$ & $13(2.9)$ & $15(3.3)$ & 0 & $2(1.5)$ & $2(1.5)$ \\
\hline NSAIDS & $4(0.9)$ & $8(1.8)$ & $12(2.6)$ & 0 & $2(1.5)$ & $2(1.5)$ \\
\hline Aspirin & $5(1.1)$ & $5(1.1)$ & $10(2.2)$ & $2(1.5)$ & 0 & $2(1.5)$ \\
\hline Alcohol & 0 & $24(5.3)$ & $24(5.3)$ & 0 & $4(3.0)$ & $4(3.0)$ \\
\hline \multirow[t]{2}{*}{ Others } & $57(12.5)$ & $39(8.6)$ & $96(21.1)$ & $15(11.4)$ & $9(6.8)$ & $24(18.2)$ \\
\hline & $255(55.9)$ & $201(44.1)$ & $456(100)$ & $85(64.4)$ & $47(35.6)$ & $132(100)$ \\
\hline
\end{tabular}

NSAIDS = non-steroidal anti-inflammatory drugs.

and 2 combined when compared with the general patient group. When comparing single and repeat presentations, a significantly greater proportion of single presentations were triaged as category 1 (OR 8.23; 95\% CI 1.05 to 64.24 , $\mathrm{p}=0.04$ ) and a significantly greater proportion of repeat presentations were triaged as categories 4 and 5 combined (OR $0.48 ; 95 \%$ CI 0.26 to $0.90, p=0.023$ ). Within the three patient groups, there were no significant differences in the proportions of patients seen outside their recommended category times ( $\mathrm{p}>0.05)$.

Overdose patients spent significantly longer in the emergency department than the general patients. Within six hours, $263(78.5 \%)$ single and $81(76.4 \%)$ repeat presentations had been managed and the patients either discharged to home or admitted to the ward (OR 1.13; 95\% CI 0.67 to $1.89, \mathrm{p}=0.65)$. This compares with $63554(94.8 \%)$ presentations from the general group (OR $0.20 ; 95 \%$ CI 0.15 to 0.26 , $\mathrm{p}<0.001$ and OR 0.18 ; $95 \%$ CI 0.11 to 0.28 , $\mathrm{p}<0.001$, respectively).

Table 3 describes the disposal of patients from the emergency department. During the study period, 23467 (35.0\%) general patients required admission to the wards or intensive care. The proportion was similar for repeat presentations (OR 0.88 ; $95 \%$ CI 0.58 to 1.32 , $\mathrm{p}=0.53$ ). Single presentations had a significantly greater proportion admitted to these wards than the general (OR 1.62; 95\% CI 1.31 to $2.01, \mathrm{p}<0.001$ ) or repeat presentation (OR $1.85 ; 95 \%$ CI 1.16 to $2.93, \mathrm{p}=0.009$ ) groups. A slightly greater proportion of repeat than single presentations required admission to the emergency department for observation (OR $0.63 ; 95 \%$ CI 0.31 to $1.26, p=0.19)$. Both groups had similar proportions of direct admissions to the psychiatric ward (OR 0.79; $95 \%$ CI 0.36 to $1.77, \mathrm{p}=0.58$ ). However, a significantly greater proportion of repeat presentations had psychiatric outpatient referrals arranged (OR $0.33 ; 95 \%$ CI 0.17 to 0.66 , $p=0.002$ ). Almost twice the proportion of single presentations were discharged home without follow up arranged (OR 1.97; 95\% CI 0.67 to $5.81, \mathrm{p}=0.22$ )

Table 4 describes the drugs and toxins used in overdose. The repeaters had a greater proportion of single drug overdoses (OR 0.70 ; $95 \%$ CI 0.47 to $1.07, \mathrm{p}=0.08$ ), paracetamol overdoses, especially paracetamol as the sole drug (OR $0.54 ; 95 \%$ CI 0.32 to 0.92 , $\mathrm{p}=0.024)$, and neuroleptic drug overdoses, especially with neuroleptics as the sole drug (OR 0.27; 95\% CI 0.11 to $0.67, \mathrm{p}=0.005$ ). Similar proportions of all other drugs were taken in overdose, although the single presenters had a slightly greater proportion of antidepressant overdoses (OR 1.19; 95\% CI 0.68 to $2.07, \mathrm{p}=0.55)$.

Five $(10.9 \%)$ repeaters and eight $(2.4 \%)$ single presenters caused self inflicted trauma, as well as overdosing, during the study period (OR $0.20 ; 95 \%$ CI 0.06 to $0.64, p=0.007)$. Three patients died in hospital as a result of their overdose, a mortality rate of $0.68 \%$. The drugs involved were paracetamol (late presentation), quinine, and a concentrated formulation of glyphosate as the isopropylamine salt (Zero). All three patients were single presenters.

The computer files of both groups of patients were examined 12 months after the study period ended. Five $(10.9 \%)$ repeaters and $15(4.5 \%)$ single presenters presented after overdose in 1995 (OR $0.38 ; 95 \%$ CI 0.13 to $1.11, \mathrm{p}=0.078$ ). Three repeaters were lost to follow up during 1995 . Of the remaining 43 $(93.5 \%)$, none had completed suicide during this 12 month follow up period.

\section{Discussion}

Ideally, patients who overdose repeatedly should be compared with those who overdose on one occasion only during their lifetime. The single presenters, as defined in this study, did not meet this criterion. The proportion of single presenters who had overdosed before the study period was not known and it is not known what proportion of single presenters 
will proceed to a "career" as a repeater. However, the study period was lengthy and it is likely that those single presenters who have or will overdose again do so relatively infrequently.

For some repeaters, parasuicidal acts tend to be clustered over a comparatively short space of time and in response to a single life event. ${ }^{14}$ The characteristics of these patients may not differ greatly from those who perform a single act under similar circumstances. This study made no attempt to discriminate between those patients who overdosed repeatedly as a result of a single life event and those who overdosed repeatedly as a result of different events or for other reasons.

The general patient presentations contained all overdose presentations. However, the overdose presentations were a small subset $(0.66 \%)$ of general presentations and comparisons between the groups should be valid. This study did not examine the patients' social class, employment or marriage status, substance or alcohol abuse, or the nature of psychiatric disease. It is recommended that these risk factors be examined in a prospective study setting.

Although an unknown number of overdose patients may never have sought medical care or may have been managed by other health care providers, it is likely that the emergency department managed the vast majority of medically significant overdoses. The small numbers of overdose patients belies their importance for two reasons. Firstly, overdose may be associated with significant suicidal ideation which requires intensive psychiatric care. However, evidence suggests that, for those patients with no immediate medical or psychiatric treatment needs on initial assessment in the emergency department, suicide within a few weeks after emergency department presentation is unusual. ${ }^{17}$ Secondly, overdose patients place a considerable strain on medical facilities. ${ }^{914}$ The higher priority triage categories, longer stays in the emergency department, and higher admission rates among overdose patients in this study, support this finding. Furthermore, overdose patients may be uncooperative, disruptive, disinhibited by alcohol or their overdose, or they may be significantly affected by adverse social circumstance or psychiatric illness. ${ }^{9-12} 1819$

Three patients died, as a consequence of their overdose, after admission to the hospital. The mortality rate for overdose of $0.68 \%$ was higher than $0.25 \%$ reported from Victoria ${ }^{5}$ and $0.4 \%$ reported from Newcastle. ${ }^{20}$ The differences are likely to be accounted for by the very small numbers of deaths.

The findings that patients in both groups were predominantly female, aged less than 35 years, and had a tendancy to present early in the week supports the findings of others. ${ }^{6} 914$ However, the repeaters were less sick medically as evidenced by their lower priority triage categories, lower admission rates to the wards and intensive care, and the finding that none of those who died were repeaters. It is assumed that the triaging was objective. Although a greater proportion of the repeat presentations had psychiatric admissions or follow up arranged from the emergency department, it cannot be interpreted that the repeaters had more apparent psychiatric illness. A greater proportion of single presenters were admitted to the hospital for medical reasons and, under present hospital guidelines, would have been referred to psychiatric services when medically recovered.

The repeaters had a higher rate of single drug overdoses, the opposite finding of others. ${ }^{21}$ Although the types of drugs ingested were similar in the two groups and similar to those reported recently, ${ }^{6223}$ the slight differences hint at the types of pre-existing psychiatric illnesses within the two groups. Antidepressants were used more frequently by single presenters and neuroleptics by repeaters. This may indicate a higher incidence of depression among single presenters and psychosis and other psychiatric disorders among repeaters. This assumes that patients overdosed on their own medications, an assumption supported by some workers ${ }^{24}$ but not by others. ${ }^{14}$ Paracetamol overdose was seen frequently. This drug is readily available and often thought to be harmless. A single paracetamol overdose may be a serious suicidal attempt or a parasuicidal gesture in patients, especially when the overdose is only a few tablets. Co-ingestion of alcohol in drug overdose has a reported incidence of $25-54 \% .^{918} 19$ The results of this study suggest that the incidence of co-ingested alcohol was low, although it is likely that its use was not consistently recorded.

It is not surprising that significantly more self inflicted trauma was seen among the repeaters. If a patient is intent on injuring and reinjuring him or herself, for whatever reason, then drug overdose is only one means. Few reports have investigated the crossover between intentional physical injury and overdose. For the same reasons, the finding that a greater proportion of repeaters presented again after overdose in 1995, is not surprising and supports the work of others. ${ }^{69132526}$

This study concludes that repeaters, as a group, are usually young females who present in the evening after a single drug overdose of benzodiazepine, paracetamol or neuroleptic, and in a stable condition medically. However, they are more likely to cause self inflicted physical injury and represent after overdose in the future. As no repeater completed suicide during the study period or the ensuing 12 months, it is concluded that the medium term suicidal risk for this group is relatively low. Although this supports the conclusions of some workers, ${ }^{13} 27$ others have found that, in a substantial proportion of cases, the suicidal act is repeated within a year and that $1-3 \%$ of those admitted for self harm commit suicide within one year. ${ }^{28}$ It is not possible to make predictions about the long term outlook for the repeaters in this study, although there seems little doubt that those who overdose repeatedly have a high long term risk of eventual suicide. ${ }^{9} 14262829$

There is a common, but mistaken, view that many people who overdose are not seriously at 
risk and that they are only making gestures or seeking attention. ${ }^{8}$ It is likely that the repeater group contains some patients at high risk of suicide and who have represented after serious, yet failed, attempts. Every overdose patient should be assessed, at an individual level, for suicidal risk and managed accordingly.

1 Harrison J, Dolinis J. Injury mortality Australia, 1993. Australian Injury Prevention Bulletin 1995;10:1-16. (Canberra: National Injury Surveillance Unit publication.)

2 Commonwealth Commonwealth Department of Health and Family Services. Here for life: a national plan for youth in distress. Canberra Australia: Commonwealth Department of Health and Family Services, 1996.

3 Commonwealth Commonwealth Department of Health and Family Services. Youth suicide in Australia: a background monograph. Canberra, Australia: Commonwealth Departmonograph. Canberra, Australia: Commonweal

4 Australian Institute of Criminology. Social factors in suicide in Australia. Trends and issues in crime and criminal justice No 52. Canberra, Australia: Australian Institute of Criminology, 1996.

5 Coleridge J, Cameron PA, Drummer OH, et al. Survey of drug related deaths in Victoria. Med J Aust 1992;157:45962 .

6 McGrath J. A survey of deliberate self-poisoning. Med J Aust 1989;150:317-24.

7 Blumenthal SI. Youth suicide: the physician's role in suicide prevention. JAMA 1990;264:3194-6.

8 Montgomery SA, Baldwin D, Green $M$. Why do amitriptyline and dothiepin appear to be so dangerous in overdose? ine and dothiepin appear to be so dangerous

9 Farmer R. Deliberate self-poisoning. Br J Hosp Med 1986; 36:437, 440-2.

10 Garvey MJ, Spaden F. Suicidal attempts in anti-social personality disorder. Compr Psychiatry 1980;21:146-9.

11 Bailey S. Critical care nurses' and doctors' attitudes to parasuicide patients. Aust J Adv Nurs 1994;11:11-17.

12 Gallop R, Lancee W, Shugar G. Residents' and nurses' perceptions of difficult-to-treat short-stay patients. Hospital and Community Psychiatry 1993;44:352-7.
13 Stocks R, Scott AI. What happens to patients who Stocks R, Scott AI. What happens to patients who
frequently harm themselves? A retrospective one-year outfrequently harm themselves? A retrospective
come study. Br J Psychiatry 1991;158:375-8.

14 Kreitman N, Casey P. Repetition of parasuicide: an epidemiological and clinical study. Br J Psychiatry 1988; 153:792-800.

15 Williams F, Ozanne-Smith J, Day L, et al. Evaluation of the implementation of the national minimum data set for injury surveillance. Melbourne: Monash University Accident Research Centre, 1995. (Report No 70.)

16 Australasian College for Emergency Medicine national triage scale. Emerg Med 1994;6:145-6.

17 Waterhouse J, Platt S. General hospital admission in the management of parasuicide. A randomised controlled trial. Br J Psychiatry 1990;156:236-42.

18 Crumley FE. Substance abuse and adolescent suicidal behaviour. JAMA 1990;263:3051-6.

19 Suokas J, Lonnqvist J. Suicide attempts in which alcohol is involved: a special group in general hospital emergency rooms. Acta Psychiatr Scand 1995;91:36-40.

20 Wynne $\mathrm{H}$, Bateman DN, Hassanyeh F, et al. Age and self-poisoning: the epidemiology in Newcastle upon Tyne in the 1980s. Human Toxicology 1987;6:511-15.

21 Owens D, Dennis M, Read S, et al. Outcome of deliberate self-poisoning. An examination of risk factors for repetition. Br J Psychiatry 1994;165:797-801.

22 Buckley NA, Whyte IM, Dawson AH, et al. Correlation between prescriptions and drugs taken in self-poisoning. Implications for prescribers and drug regulation. Med J Aust 1995;162:194-7.

23 Buckley NA, Whyte IM, Dawson AH, et al. Self-poisoning in Newcastle, 1987-1992. Med J Aust 1995;162:190-3.

24 Stenager EN, Jensen K. Attempted suicide and contact with the primary health authorities. Acta Psychiatr Scand 1994; 90:109-13.

25 Hassanyeh F, O'Brien G, Holton AR, et al Repeat self-harm: an 18-month follow-up. Acta Psychiatr Scand 1989;79:265-7.

26 Barnes RA. The recurrent self-harm patient. Suicide Life Threat Behav 1986;16:399-408.

27 Rodger CR, Scott AI. Frequent deliberate self-harm: repetition, suicide and cost after three or more years. Scott Med J 1995;40:10-12.

28 Kreitman N. Parasuicide. London: Wiley, 1977.

29 Pearce CM, Martin G. Predicting suicide attempts among adolescents. Acta Psychiatr Scand 1994;90:324-8. 